\title{
Glucose-Powered Pulsatile Release
}

By Yasuhiro Nishii, ${ }^{a, b}$ Swapnil Gandhi, ${ }^{a}$ and Eric Nuxoll ${ }^{a, *}$

${ }^{a}$ Department of Chemical and Biochemical Engineering

4133 Seamans Center for the Engineering Arts \& Sciences

University of Iowa

Iowa City, IA 52242, U.S.A.

${ }^{\mathrm{b}}$ Department of Applied Chemistry and Biotechnology

Niihama National College of Technology

Ehime, 792-8580, Japan

*corresponding author

email: eric-nuxoll@uiowa.edu

office: 1-319-353-2377

fax: $1-319-335-1415$

Keywords. Controlled Release; Pulsatile Drug Delivery; Hydrogel; Glucose Oxidase; Reactive Membranes. 


\begin{abstract}
.
Pulsatile release is required for many drugs from hormones to vaccines, but automating this release typically requires elaborate pumping systems with electronic controls and transcutaneous catheters. This paper describes a materials-based approach to this functionality, using a combination of composite polymer membranes to provide discrete pulses of drug at preprogrammed intervals using only a static concentration of glucose in water. Drug is encapsulated in acid-sensitive polymer membranes. Enzymes which convert glucose to acid are immobilized in other polymer membranes, along with sacrificial acid scavengers. These membranes are stacked alternately into a polymer laminate and sealed around the bottom and edges. Glucose diffuses steadily into the top membrane and is converted to acid. This acid is consumed by the scavenger until the scavenger is exhausted, at which point it triggers the acidsensitive drug membrane below. That membrane swells and delaminates from the stack, releasing its payload, and the process repeats, potentially with different drugs in each layer and different preprogrammed delay times between each dose. This paper provides the first experimental demonstration of such a system, characterizes it components, models the system computationally, and provides basic design rubrics for further development.
\end{abstract}




\section{Introduction.}

Many chemicals, particularly pharmaceuticals, are best administered in discrete pulses. In some cases, this is due to chronobiological factors, where physiological response is different depending on the time of day, month, or year. For instance, timing the administration of cancer therapies to the patients' circadian rhythyms has been shown to improve outcomes $(1,2)$. In other cases, the physiological response may change with duration of exposure, as demonstrated by the induction of tolerance to opiates(3) and nitrate(4) therapies. Periodic challenges to the immune system are used both for induction of tolerance to allergens(5) and for induction of sensitivity to pathogens $(6,7)$. For reproductive hormones, pulsatility is often a critical component of the signal(8-10), and the pulsatility of parathyroid hormones determines whether bone is remodeled or simply resorbed $(11,12)$. Where multiple chemicals are needed, their sequence of application may be critical, from angiogenesis(13) to insect control(14).

The simplest way to achieve pulsatile delivery is manual administration of individual pills or injections. Manual application is often problematic, however, for a variety of reasons ranging from simple forgetfulness to fear of needles $(15,16)$ to limited access to healthcare providers. Automation of pulsatile delivery, on the other hand, typically requires pumps, power supplies, circuitry, central reservoirs, and chronic transcutaneous conduits. Materials-based approaches to pulsatile delivery have been limited largely to capsules which release at a predetermined time based on degradation or osmotic swelling of the capsule(17-19). Several systems have demonstrated single delayed pulses with this approach, but none have demonstrated more than three discrete pulses from a single administration, as slight errors in timing control quickly lead to broadened or overlapping pulses. 
By linking the release of one pulse to the release of the next, this problem can be overcome as depicted in Figure 1. Each dose of drug is embedded in a stimuli-sensitive depot membrane. These depot membranes are stacked between barrier membranes which each block the stimulant for a fixed period of time. Once a barrier membrane is exhausted, the stimulant triggers the release of drug dose from the underlying depot, then begins penetrating the next barrier. In this manner, multiple distinct pulses can be obtained from a single polymer chip. Each pulse may contain a different drug and may be separated from the previous pulse by a different, predetermined delay time. In its simplest form, soluble drug is layered between degradable polymer barriers. As each barrier erodes away, the underlying layer of drug is

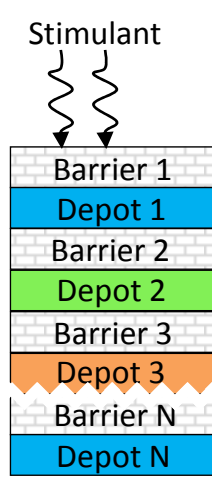

Time $<\mathrm{T}_{1}$

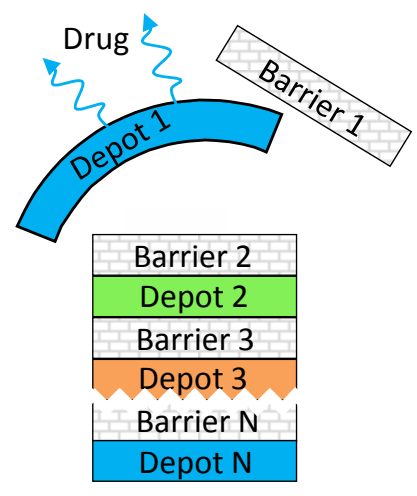

Time $=\mathrm{T}_{1}$

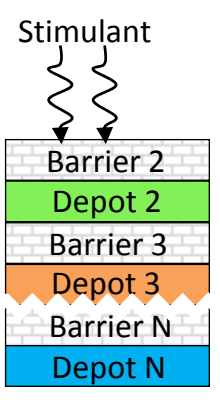

$\mathrm{T}_{1}<$ Time $<\mathrm{T}_{2}$

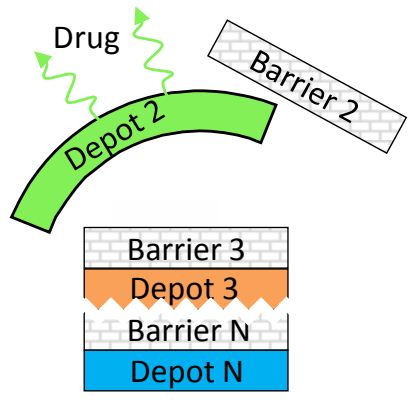

Time $=\mathrm{T}_{2}$

Figure 1: Schematic of Barrier-mediated Pulsatile Release. Stimulant is blocked by Barrier 1 until time $T_{1}$, as shown in the first panel. Once Barrier 1 is exhausted, stimulant triggers release from Depot 1 and begins penetrating Barrier 2. At time $\mathrm{T}_{2}$, Barrier 2 is exhausted, prompting a pulse of drug from Depot 2. The process continues until all doses are released.

released and the next barrier starts to degrade(20-23).

Relying on polymer degradation for pulse timing is problematic, however, because it requires the polymer layer to be completely surface-eroding-i.e., the polymer layer must get uniformly thinner while still completely protecting the depot until suddenly the entire layer disappears. Most degradable polymers are bulk-eroding, and doubling the delay time requires 
more than doubling the thickness of the barrier. Recently, this approach has been generalized using stimuli-sensitive hydrogel depots $(24,25)$. When exposed to acid, the hydrogel membranes swell with water, allowing the solid drug within to dissolve and release. The acid is blocked for a specific period of time by barrier membranes loaded with nanoparticulate zinc oxide, an acid scavenger. The delay time scales linearly with both the scavenger loading and the square of the barrier membrane thickness. Up to ten discrete pulses have been demonstrated from these passive polymer chips, with no apparent limit on the maximum number of $\operatorname{pulses}(24)$.

For physiological applications, however, constant acid sources are unavailable except in the digestive tract, which has a relatively short residence time. Glucose, however, is distributed throughout the body via the bloodstream in a relatively tight, well-regulated concentration range of $70-140 \mathrm{mg} / \mathrm{dL}$. This paper reports the creation of a polymer laminate which releases discrete pre-timed pulses of drug in response to a constant glucose source. Development and characterization of constituent materials are followed by construction and demonstration of prototype devices, then in silico investigation of key design parameters.

\section{Materials and methods}

\subsection{Materials.}

Methyl methacrylate (MMA), 2-dimethylamino ethyl methacrylate (DMA), and divinyl benzene (DVB) were obtained from Sigma-Aldrich (Milwaukee, WI). Polymerization inhibitors in these monomers were removed by adsorption on alumina (Fisher Scientific, Pittsburgh, PA), the monomers were then refrigerated until used. 2, 2'-Azobisisobutyronitrile (AIBN) powder and D-gluconic acid (51\% aqueous solution) were obtained from Sigma Aldrich (St. Louis, MO) and refrigerated until use. Zinc oxide ( $\mathrm{ZnO})$, citric acid, sodium 
hydroxide, and potassium chloride were obtained in crystalline powders from Sigma-Aldrich and used as received. Glucose oxidase (type X-S) and catalase were obtained from SigmaAldrich as lyophilized powders and stored at $-18{ }^{\circ} \mathrm{C}$ until used. Monobasic potassium phosphate and sodium chloride were used as received from Research Products International (Mt. Prospects, IL). Poly (vinyl alcohol) (PVA, 99\% hydrolyzed, MW 133,000) was obtained from Polysciences (Warrington, PA) and used as received. Methylene blue (MB, SigmaAldrich) and methyl orange (MO, Alfa-Aesar, Wardhill, MA) were ground with a mortar and pestle then screened at 100 mesh before use.

\subsection{Depot membranes.}

\subsubsection{Depot membrane fabrication.}

Depot membranes were created by the copolymerization of methyl methacrylate (MMA) and dimethylamino ethyl methacrylate (DMA) in a 72:28 molar ratio of MMA:DMA with $0.1 \% \mathrm{w} / \mathrm{w}$ divinyl benzene (DVB) crosslinker. Poly(methyl methacrylate) (PMMA) is used in a wide range of commercial and industrial applications due to its excellent mechanical properties, including its glassy, hydrophobic nature, which makes it effectively impervious to water. Incorporation of an amine-containing comonomer, however, allows ionization of the polymer at low $\mathrm{pH}$, making the polymer hydrophilic and prompting it to take up five times its own weight in water. Several amine comonomers have been investigated, with DMA providing the strongest swelling response to acidic $(\mathrm{pH}<6)$ environments $(26,27)$. By incorporating comonomers or cross-linking agents which can be cleaved hydrolytically or enzymatically, biodegradable pH-sensitive PMMA gels have also been produced(28).

Fabrication of the depot membranes adapted protocols used previously for acidtriggered BMPR systems(24). Poly(methyl methacrylate-co-dimethylamino ethyl 
methacrylate) (PMMA/DMA) membranes were synthesized by heating 6.315 mL MMA, 3.909 mL DMA, 10.5 $\mu \mathrm{L}$ DVB crosslinker, and $48.03 \mathrm{mg}$ AIBN initiator to $70{ }^{\circ} \mathrm{C}$ with stirring until the solution reached a viscosity of approximately $65 \mathrm{cP}$. The solution was then chilled quickly to room temperature before adding the payload chemical to be released. This prepolymerization step reduces the effect of polymerization inhibition by payload chemicals such as methylene blue (MB)(29) as well as the effect of the negative volume of polymerization in a closed system. Methyl orange (MO) or MB powder were dispersed into the oligomer solution by stirring and sonication (Cole Parmer CPX750 with $3.2 \mathrm{~mm}$ microtip) at $300 \mathrm{~W}, 20$ $\mathrm{kHz}$ for $1 \mathrm{~min}$. The suspension was then poured between a pair of silanized glass plates with poly(tetrafluoroethylene) (PTFE) spacers to control the membrane thickness. The clamped plates were mounted vertically in a vacuum oven under nitrogen at $60{ }^{\circ} \mathrm{C}$ for 18 hours, then removed, cooled, removed from the glass plates, and stored under ambient conditions until used.

\subsubsection{Depot membrane characterization.}

The swelling kinetics and equilibrium swelling ratio of the depot membranes were measured in sodium gluconate buffers at $\mathrm{pH}$ values of 3.0, 3.5, 4.0, 4.5, 5.0, 5.5, and 6.0. Disks of PMMA/DMA (without payload chemicals) approx. $130 \mu \mathrm{m}$ thick and $15 \mathrm{~mm}$ wide were immersed in $500 \mathrm{~mL}$ solutions of gluconate buffer with a buffer strength (gluconic acid + gluconate anion) of $0.01 \mathrm{M}$ and a total ionic strength of $0.1 \mathrm{M}$ obtained by addition of sodium chloride. The disks were periodically removed from solution, blotted, dry, and weighed. Their thickness was measured by micrometer before re-immersion in solution.

The release rate and the effect of membrane thickness were investigated at $\mathrm{pH} 3$ and 7 using more permeable weak-acid buffers. PMMA/DMA disks containing 5\% w/w MO with 
thicknesses of $190 \mu \mathrm{m}$ and $320 \mu \mathrm{m}$ were immersed in $500 \mathrm{~mL}$ solutions of sodium citrate $(\mathrm{pH}$ 3) or phosphate $(\mathrm{pH} \mathrm{7)}$ buffer solution. The membranes were periodically reweighed to determine swelling, while MO release was monitored with a UV/Vis spectrophotometer (Cary 50, Varian Inc., Mulgrave, Austrailia) at $461 \mathrm{~nm}(\mathrm{pH}$ 7) or $505 \mathrm{~nm}(\mathrm{pH} 3)$.

The partition coefficient of MO in PMMA/DMA was estimated by sequentially adding 30-mg pieces of depot membrane (5\% MO by weight) into $100 \mathrm{~mL}$ of $\mathrm{pH} 3$ gluconate buffer with a buffer strength of $0.01 \mathrm{M}$ and an ionic strength of $0.1 \mathrm{M}$. Once the $\mathrm{MO}$ concentration in the well-stirred buffer plateaued, as determined by periodic UV/Vis measurement, then the next depot membrane sample was added. Four samples were added in total.

Depot membranes with larger DMA mol fractions were also investigated. Membranes with MMA:DMA ratios of 1:99, 10:90, 20:80, and 50:50 were synthesized. Their swelling behavior was investigated in gluconate buffers of various $\mathrm{pH}$ as well as neutral saline solution.

\subsection{Barrier membranes}

Barrier membranes were fabricated using poly(vinyl alcohol) (PVA), a pH-insensitive biocompatible polymer which forms a highly permeable hydrogel. By immobilizing zinc oxide nanoparticles in a PVA matrix, barrier membranes have already been demonstrated which selectively block acid transport for a fixed period of time before allowing rapid transport of both the acid stimulant and the payload chemical(30,31). Besides blocking the stimulant for a prescribed period of time, the barrier membranes for the present system must also transduce the stimulant, converting glucose into the acid that triggers depot swelling.

To create a membrane that would perform this transduction, glucose oxidase and catalase were dissolved in a $7.5 \mathrm{wt} \%$ solution of poly(vinyl alcohol) (PVA) at loadings of 0.1 mg / mg PVA and $0.03 \mathrm{mg} / \mathrm{mg}$ PVA, respectively. The solution was poured on a PTFE plate 
at room temperature and calendared with a custom-built doctor blade, then dried under ambient conditions. A $0.029 \mathrm{~g}$ sample of the dry $80 \mu \mathrm{m}$ thick membrane was placed in a $14 \mathrm{~mL}$ sample of $150 \mathrm{mM}$ saline containing $6.67 \mathrm{mM}(120 \mathrm{mg} / \mathrm{dL})$ glucose. When the $\mathrm{pH}$ of the solution dropped to 3.2 the membrane was transferred to a fresh $15 \mathrm{~mL}$ saline-glucose solution; this transfer was repeated, using a total of three $15 \mathrm{~mL}$ aliquots. The process was then repeated with membrane which had been heat treated at $150{ }^{\circ} \mathrm{C}$ under nitrogen for two hours to crosslink the PVA for better enzyme immobilization. The minimum solution $\mathrm{pH}$ for these trials was 2.9.

Membranes with barrier functionality were fabricated as described previously $(24,30)$. $\mathrm{ZnO}$ nanoparticles were distributed in an $15 \% \mathrm{w} / \mathrm{w}$ suspension via stirring and sonication, then stirred into the PVA solution to achieve the desired $\mathrm{ZnO}$ content. The mixture was then cast on a PTFE plate, calendared to the desired thickness, dried under ambient conditions, and heattreated between two glass plates at $150{ }^{\circ} \mathrm{C}$ under nitrogen for 2 hours to crosslink. Membranes were stored dry, then hydrated for at least 24 hours prior to use.

The transport properties of $\mathrm{MO}$ and $\mathrm{MB}$ in both PVA barrier membranes and PMMA/DMA depot membranes were investigated previously. To determine the gluconic acid permeability of crosslinked PVA barrier membranes, an individual membrane was mounted between two well-stirred $20 \mathrm{~mL}$ diaphragm cells of $100 \mathrm{mM}$ saline. One $\mathrm{ml}$ of $0.1 \mathrm{M}$ gluconic acid was spiked into the upstream cell and the $\mathrm{pH}$ of both cells were monitored over time. The process was repeated using a laminate of four samples cut from the same membrane, effectively increasing the membrane thickness by a factor of four.

\subsection{Device Fabrication.}

BMPR devices were fabricated by stacking barrier and depot membranes in alternating order on a $1.1 \mathrm{~mm}$ thick, $15 \mathrm{~mm}$ wide PTFE disk within a $3.0 \mathrm{~cm}$-wide mold. Hot wax is 
poured around and above the stack in the mold, then frozen. The device is then removed from the mold and inverted, and the PTFE disk is removed. The PTFE disk served as a temporary spacer to recess the top of the stack from the top of the wax sidewall and prevent premature delamination of the device. Because the hydrated PVA barrier membranes have poor adhesion to the wax sidewall, a payload-free PMMA/DMA depot membrane is stacked in front of the first barrier layer (i.e., in contact with the PTFE disk) to ensure that the top layer of the stack is completely sealed to the sidewall. The PVA barrier layers are also $1 \mathrm{~mm}$ wider than the 15 mm-wide PMMA/DMA depot layers to provide better adhesion to the sidewall.

Conventional BMPR devices containing two 5\% MB w/w depots were constructed with barriers containing either $10 \%$ or $30 \% \mathrm{w} / \mathrm{w} \mathrm{ZnO}$ in front of each pulse. Similar devices using barrier membranes containing $10 \% \mathrm{w} / \mathrm{w}$ enzyme were also constructed, though subsequent testing indicated that the MB payload binds strongly to the enzyme, preventing its release. Subsequent devices were constructed using depots with $5 \% \mathrm{w} / \mathrm{w}$ MO in a 4-pulse construction. In these devices, each barrier membrane consisted of two separate layers: a non-heat-treated enzyme-loaded PVA layer adjacent to the underlying depot and a heat-treated PVA layer loaded with $\mathrm{ZnO}$ nanoparticles. Layers containing $0 \%$ or $10 \% \mathrm{ZnO}$ were incorporated into separate devices. For these devices, the top payload-free PMMA/DMA membrane and first ZnO-loaded PVA layer were omitted.

\subsection{Device Characterization.}

The 2-pulse MB-loaded devices were first immersed in $300 \mathrm{~mL}$ of well-stirred $\mathrm{pH} 7$ sodium phosphate buffer for 16 hours to confirm their inactivity at neutral $\mathrm{pH}$. They were then immersed in $1000 \mathrm{~mL}$ of $\mathrm{pH} 3$ sodium citrate buffer. The time profile of MB release was 
tracked by UV/Vis scanning via fiber-optic probe. Buffer $\mathrm{pH}$ was automatically logged to ensure stability at $\mathrm{pH} 3$.

The 4-pulse MO-loaded devices were immersed in $100 \mathrm{~mL}$ of $100 \mathrm{mM}$ saline containing $100 \mathrm{mM}$ glucose. The MO concentration of the solution was tracked by UV/Vis scanning, along with automatic tracking of the solution $\mathrm{pH}$.

\subsection{In silico investigation.}

The primary motivation for using glucose as the stimulant for BMPR systems is drug delivery, where the bloodstream provides a well-regulated endogenous glucose source. While mimicking the physiological source condition can be achieved in vitro simply by adding more

glucose as needed, mimicking physiological sink conditions is much more difficult. If gluconate ions build-up in the sink, they decrease the dissociation of gluconic acid, effectively buffering the system and preventing the hydrogel depots from swelling. Without a means to selectively remove all the gluconate being generated by the enzymes, the only way to avoid their build-up is to continually flush the system with a large flow of fresh glucose solution. Besides the obvious problems with managing such large amounts of solution, this also strongly dilutes the concentration of released chemical, making its release rate much more difficult to accurately quantify.

Earlier investigations of non-delaminating BMPR systems prompted the development of a computational model to investigate design parameters not readily accessible experimentally(25). This model was extended to include dynamic stimulant transformation and accommodate selective sequestration of the enzyme within the barrier membrane. The model uses the centered finite difference method and explicit Euler method to calculate Fickian diffusion in the barrier membranes. In the depot membranes, flux is calculated directly from 
node-to-node concentration differences in order to account for acid flux into depot nodes still in their shrunken state - the hydrogel is assumed to be binary, existing only in its fully swollen or fully shrunken state at each node. At each time step, transport calculations are followed by reaction calculations: converting glucose to acid based on local enzyme and glucose concentrations, consuming acid based on scavenger presence, and maintaining drug saturation by dissolution of local solid drug. 60 barrier nodes and 120 depot nodes were used with a $4 \times 10^{-}$ ${ }^{4} \mathrm{~s}$ time step based on prior model validation. The complete FORTRAN code is provided in the Supplementary Materials.

Three key design variables were investigated computationally: barrier thickness, enzyme sequestration, and total enzyme activity. Devices with barrier membranes exposed to a physiological glucose concentration $(0.005 \mathrm{M}=90 \mathrm{mg} / \mathrm{dL})$ were modeled with PVA barrier membranes ranging in thickness from 50 to $500 \mu \mathrm{m}$ with an average enzyme activity concentration of $2 \times 10^{-7} \mathrm{M} / \mathrm{s}$. This activity was distributed across different regions of the barrier thickness. For instance, in some models all of the enzyme activity was sequestered to the $10 \%$ of the barrier closest to the depot, so no glucose is converted until it has diffused across $90 \%$ of the barrier. Similarly, trials sequestering the enzyme in the closest $20 \%$ of the barrier, closest $30 \%, 40 \%$, etc. were performed, up to trials where the enzyme was homogenously distributed across the barrier. All other parameters were based on results from the characterization studies. These trials indicated that the system was glucose-limited; subsequent trials investigated systems with an average enzyme activity concentration of $2 \times 10^{-8} \mathrm{M} / \mathrm{s}$, again looking at the effect of barrier thickness and enzyme concentration, with a lower DMA concentration in the depot to facilitate quicker release times. Finally, these systems were modeled with $10 \% \mathrm{w} / \mathrm{w}$ $\mathrm{ZnO}$ to induce a lag time prior to release. 


\section{Results and Discussion.}

\subsection{Depot membranes.}

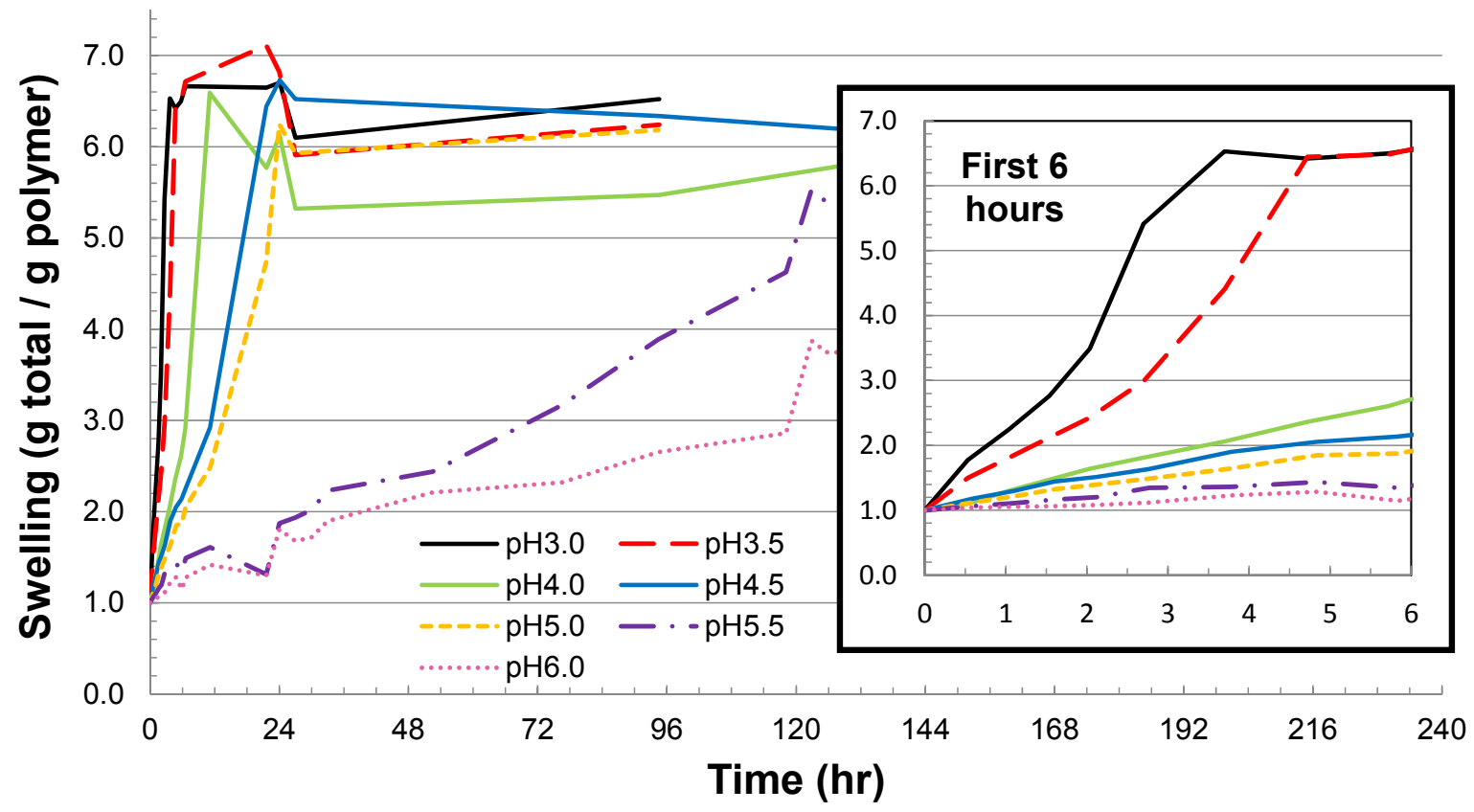

Figure 2: pH-sensitivity of PMMA/DMA. PMMA/DMA disks immersed in gluconic acid buffer at various $\mathrm{pH}$ swell at different rates, with increasing proportions of protonated acid prompting faster swelling.

The swelling behavior of PMMA/DMA depot membranes in gluconate buffer at several $\mathrm{pH}$ values is shown in Figure 2 . With $0.1 \%$ by mol crosslinker, the equilibrium swelling ratio of the gel (g water / $\mathrm{g}$ dry polymer) is approximately 5 at $\mathrm{pH} 3$, consistent with studies in other weak-acid buffers. The swelling rate, however, is markedly slower in gluconate buffer than in citrate buffer. Previous studies have shown that under most conditions, the swelling rate of PMMA/DMA is limited by stimulant transport and that the limiting stimulant species is not hydronium ions, but rather the neutral weak $\operatorname{acid}(24,32)$. This is demonstrated in Figure 2, where the hydronium ion concentration decreases by a factor of $10^{0.5}$ from one buffer to the next, but the swelling rate changes by a different factor in each case. From $\mathrm{pH} 3$ to $\mathrm{pH} 3.5$, the concentration of neutral gluconic acid decreases only $30 \%$, and the time required to reach 
equilibrium swelling increases by about $30 \%$. At larger $\mathrm{pH}$ values, the neutral gluconic acid concentration drops more rapidly, decreasing by a factor of 8 from $\mathrm{pH} 4.5$ to 5.5 , while the time required to reach equilibrium swelling increases by roughly a factor of 8 .

While previous studies using citrate buffers demonstrated the same fundamental behavior, PMMA/DMA membranes took approximately five times longer to reach their equilibrium swelling ratio in gluconate buffer than in citrate buffer at each $\mathrm{pH}$ value, with identical buffer strengths and ionic strengths. This is particularly noteworthy as the neutral acid concentration, whose transport dictates the swelling rate, is over three times larger in gluconate buffers than in citrate buffers at the same $\mathrm{pH}$ and buffer strength, due to gluconic acid's larger $\mathrm{pKa}$ value $(3.62$ vs. 3.13)(33). This suggests that gluconic acid permeability in shrunken PMMA/DMA is approximately fifteen times smaller than citric acid permeability.

To decouple the swelling rate from the investigation of release rate, $\mathrm{pH}$ 3 citrate buffer was used. Figure 3 shows the swelling and release of $\mathrm{MO}$ from
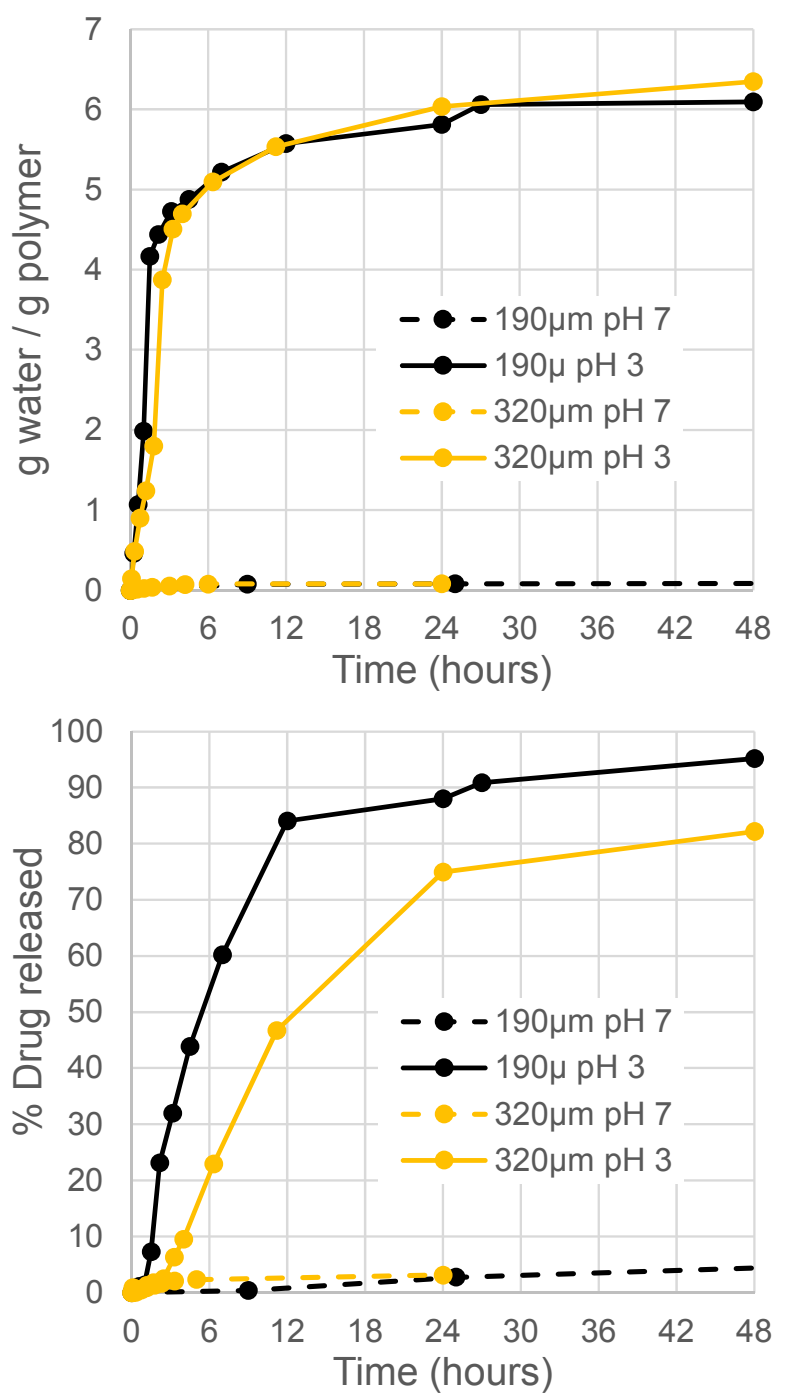

Figure 3: Swelling and solute release from PMMA/DMA hydrogels. PMMA/DMA disks immersed in gluconic acid buffer at $\mathrm{pH} 3$ swelled rapidly, but released more slowly, showing a thickness dependence characteristic of diffusion-limited release. Solute release at $\mathrm{pH} 7$ was negligible. 
PMMA/DMA depot membranes at two different thicknesses. In $\mathrm{pH} 7$ phosphate buffer, no swelling or MO release is observed. In $\mathrm{pH} 3$ citrate buffer, however, membranes at both thicknesses promptly swell and begin releasing their payload. The release of MO is significantly slower than the swelling rate, however, due in part to the low steady-state permeability of MO in swollen PMMA/DMA hydrogels (approximately $3 \times 10^{-7} \mathrm{~cm}^{2} / \mathrm{s}$ ) and due to negatively charged MO's propensity for ion exchange within the positively charged, swollen hydrogel. The transport limitation (including transient resistance due to ion exchange) to the release rate is confirmed by the fact that it takes nearly three times longer for the MO to release from the membrane which is approximately $3^{0.5}$ times thicker.

The most critical feature of Figure 3 is the swelling and payload release (or lack thereof) at $\mathrm{pH}$ 7. At neutral $\mathrm{pH}$ the depot membrane remains completely glassy and hydrophobic, encapsulating the solid payload in a solvent-free environment and completely blocking its release. Many other polymers have been investigated for their stimuli-sensitivity, most notably pH-sensitive copolymers of N-isopropylacrylamide (PNIPA)(34), but even in its shrunken state, PNIPA remains rubbery and partially hydrated, allowing premature dissolution and release of the payload. Previous studies of PMMA/DMA copolymers investigated copolymers with MMA:DMA molar ratios ranging from 100:0 to 70:30, showing increasing equilibrium swelling and swelling rate with increasing DMA content. In this study, DMA contents of 50, 80,90 , and $99 \%$ by mol were also investigated. The trend of increasing equilibrium swelling and swelling rate at low $\mathrm{pH}$ continued with 50\% DMA hydrogel swelling to 15 times its dry weight in an hour in gluconic acid at $\mathrm{pH} 3$, while 99\% DMA hydrogel swelled beyond mechanical stability almost immediately. However, while the swelling of 50\% DMA hydrogel 
in neutral deionized water was below quantification, it did exhibit persistent leaching of its MB payload. Larger DMA contents exhibited rapid, significant swelling at neutral $\mathrm{pH}$.

\subsection{Barrier membranes.}

PVA is an attractive barrier membrane matrix for several reasons. It is already approved for blood-contacting applications, it is relatively inert and $\mathrm{pH}$ insensitive, it can be cast from a resin without need to leach out residual monomers, at it forms a hydrogel with a large permeability for both stimulant and payload. Diaphragm cell trials determined that the membrane's permeability to gluconic acid in saline - in the absence of a reactive scavenger-is $7.04 \times 10^{-6} \mathrm{~cm}^{2} / \mathrm{s}$ (st.dev. $\pm 1 \%$ ). This high permeability permits rapid activation of the underlying hydrogel depot once the reactive scavenger is depleted. The breakthrough time for reactive barrier membranes, $t_{L}$, is given in Equation 1, where $\ell$ is the membrane thickness, $D$ and $H$ are the stimulant's diffusion and partition coefficients, respectively, $C_{\text {stim }}$ is the stimulant concentration in the external source and $C_{\text {scav }}$ is the stoichiometrically equivalent concentration of scavenger in the membrane.

$$
t_{L}=\frac{\ell^{2}}{6 D}\left(1+\frac{3 C_{\text {scav }}}{H C_{\text {stim }}}\right)
$$

(Equation 1)

While a large stimulant permeability $D \times H$ does reduce the breakthrough time, it can be easily compensated by increasing the scavenger loading or especially by increasing the barrier thickness, as the breakthrough time is proportional to the square of the membrane thickness.

Immobilization of enzymes in PVA has also been previously studied. Non-crosslinked PVA does not appear to interfere with the activity of the enzymes immobilized in it, though without crosslinking the enzymes are not truly immobilized. Dissolution trials on $120 \mu \mathrm{m}$ PVA membranes in stagnant room-temperature deionized water indicated a mass loss of $48 \%$ in 24 hours. Enzyme immobilized within such a dissolving matrix will leach into the surrounding 
medium, as shown in Figure 4.

With each transfer to fresh

glucose solution, the membrane

showed a significant drop in

enzyme activity. Previous studies

on enzyme immobilization in

PVA membranes indicated that

chemical crosslinkers will react

with the enzyme as well as the

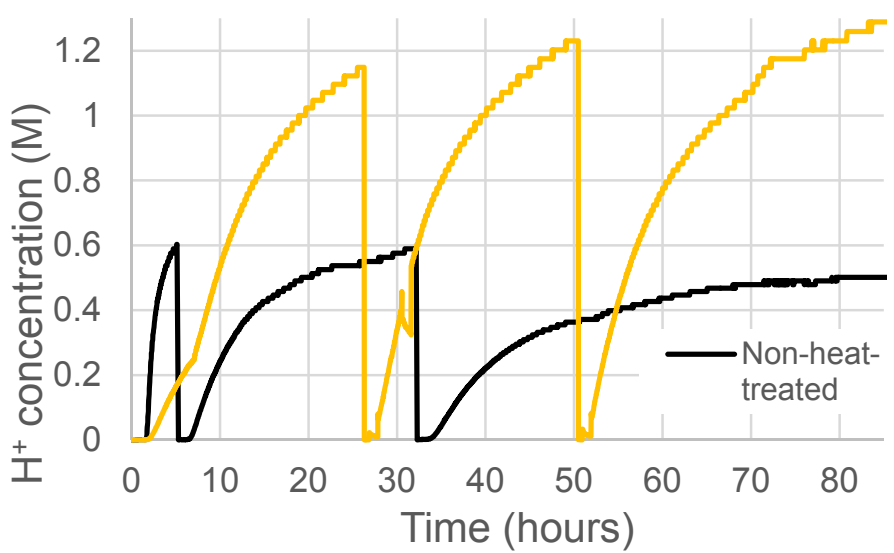

Figure 4: Enzyme immobilization in PVA hydrogels. PVA hydrogel loaded with glucose oxidase enzyme decreased in enzymatic activity after transfer to subsequent glucose solutions, indicating loss of enzyme from the polymer matrix. After heattreatment at $150{ }^{\circ} \mathrm{C}$, the hydrogel loses $2 / 3$ of its initial enzyme activity, but the remaining active enzyme is efficiently retained across multiple transfers.

PVA, compromising its

activity(35). Moreover, chemical crosslinking often involves an acid catalyst which would be incompatible with any acid scavengers loaded in the membrane. Physical crosslinking through freeze/thaw cycling, on the other hand, did not appear to decrease enzyme activity, but did allow enzyme leaching(35). To achieve the complete immobilization of chemical crosslinking without adding a chemical crosslinker, this study pursued an alternative crosslinking strategy. Previous reports have shown that dry PVA membranes cured at $150{ }^{\circ} \mathrm{C}$ become completely insoluble and exhibit an increase in $\mathrm{C}-\mathrm{O}$ bonds as indicated by infrared spectrometry(36), presumably condensing nearby pendant hydroxyl groups to form ether linkages between chains. Dissolution trials on membranes cured at $150{ }^{\circ} \mathrm{C}$ for two hours under nitrogen demonstrate no mass loss after several days in well-stirred DI water at temperatures up to $50{ }^{\circ} \mathrm{C}$. At higher temperatures, however, the membranes quickly dissolve, indicating that the crosslinking is thermally reversible. Applying this treatment to the enzyme-loaded PVA membranes and repeating the activity trial, Figure 4 shows that while the enzyme's activity was reduced by 67 
$\%$ after crosslinking, all of the remaining enzyme activity was retained as the membrane was moved from one fresh glucose solution to the next.

\subsection{Experimental BMPR Devices}

To demonstrate the BMPR concept, enzyme-free devices containing two doses of MB were constructed. Each dose in the first device, depicted at the top of Figure 5, was protected by a reactive barrier membrane approximately $170 \mu \mathrm{m}$ thick containing $30 \%$ by weight $\mathrm{ZnO}$ nanoparticles. In the second device, the first barrier membrane is changed to a $68 \mu \mathrm{m}$ thick barrier containing only $10 \%$ by weight $\mathrm{ZnO}$. After immersion in $\mathrm{pH} 3$ buffer, the release profiles shown in Figure 5 demonstrate two sharp pulses at timings within $10 \%$ of those predicted by Equation 1 . 

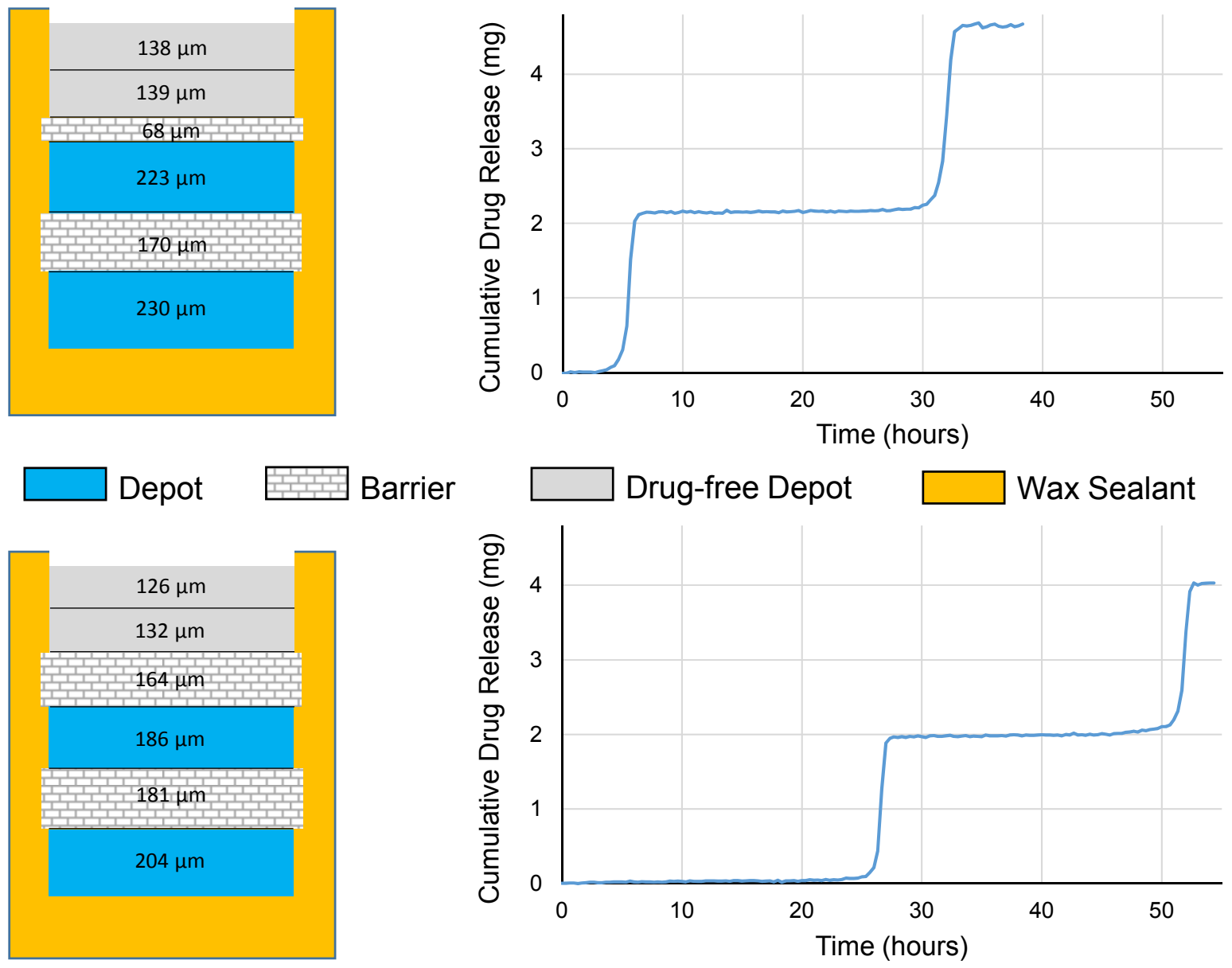

Figure 5: BMPR release controlled by barrier thickness. At left, schematics of BMPR devices with $170 \mu \mathrm{m}$ barriers. When the thickness of one of the barriers is reduced from 170 to $70 \mu \mathrm{m}$ (top), the delay time of the pulse decreases with the square of the decrease in barrier thickness. Note that the vertical and horizontal length scales of the schematics are different for clarity. The actual device aspect ratio resembles a coin's.

Glucose-triggered release is much more complex, however. To maximize enzyme 
activity while maintaining enzyme immobilization within the device, a bilayer barrier membrane system was developed as depicted in Figure 6. Enzyme was immobilized within a non-crosslinked layer of the barrier membrane, placed adjacent to its corresponding depot. The outer face of the barrier membrane consisted of crosslinked PVA which prevented any of the enzyme from escaping before the underlying depot had swollen and prompted delamination. This system released no drug in a sink of glucose-free saline. Upon spiking the sink to $0.1 \mathrm{M}$ glucose, however, the system began releasing its MO payload, as shown in Figure 6.

Figure 6 also demonstrates the versatility of this approach. The barrier membranes in this device did not contain any scavenger, so the stimulant was able to proceed quickly from one depot to the next, prompting continuous release. While the release from a single large
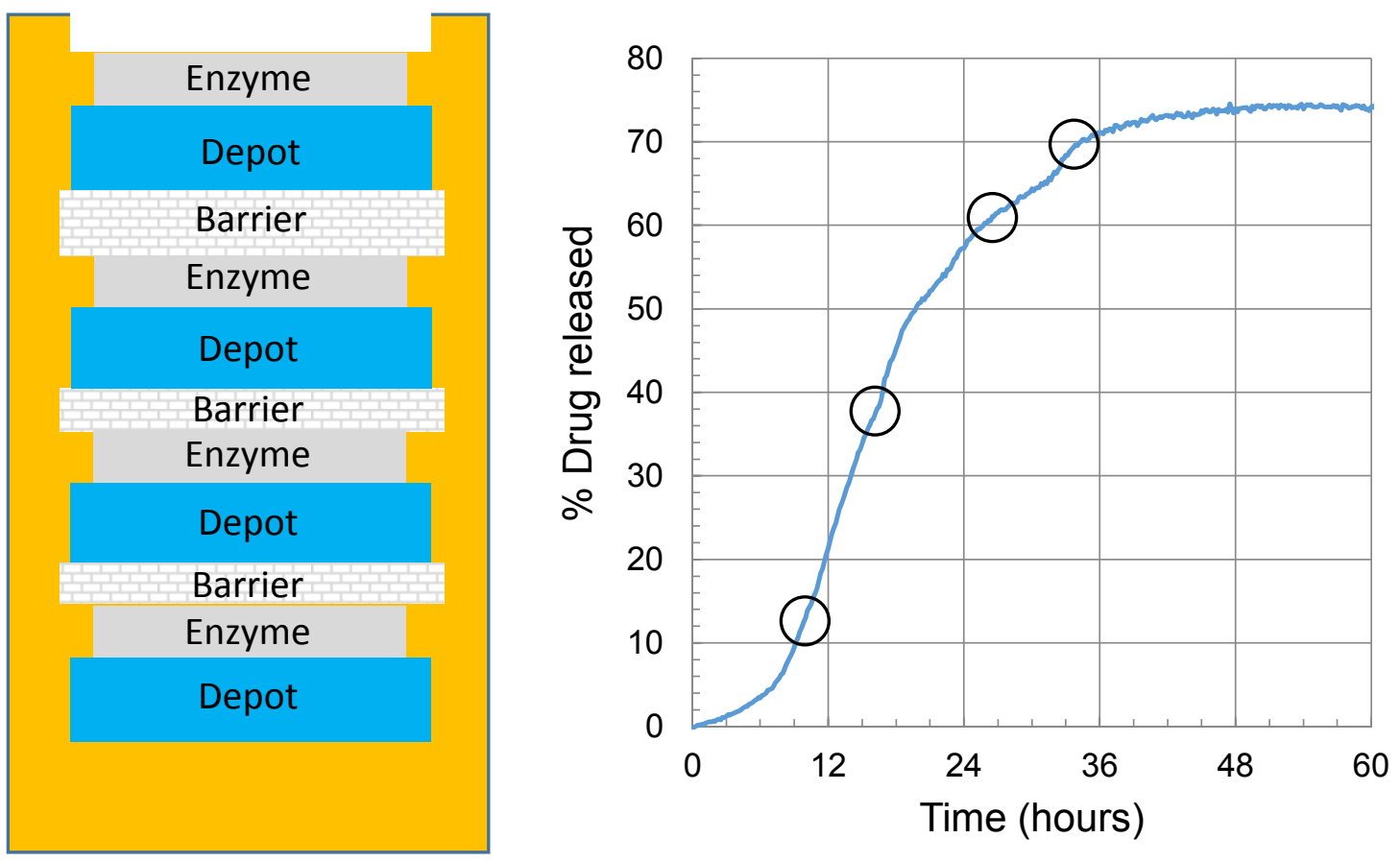

Figure 6: Sustained release triggered by glucose. At left, schematic of a BMPR devices with $\mathrm{ZnO}$-free barriers. Without any base to scavenge the produced acid, the depots release sequentially with little delay time in between. This provides an essentially sustained-release functionality. Circles on the chart at right indicate times when depots delaminated from the device. Note that the vertical and horizontal length scales of the schematics are different for clarity. The actual device aspect ratio resembles a coin's. 
depot would decay quadratically over time as solute must escape from deeper within the depot, in a BMPR system the release rate can be constantly renewed, providing steady, continuous release. The release in Figure 6 decays only as the concentration in the sink gets large enough to restrict partitioning out of the device; the release from the later pulses is significantly less than the release from the first pulse, and overall only $3 / 4$ of the MO is released.

This is due to an important limitation in the current experimental system. In order for the hydrogel depot to be swollen by organic acids, it must accept acid protons to become charged and hydrophilic. Negatively charged solutes, then, will be electrostatically attracted to the matrix, strongly hindering their release. Positively charged solutes such as MB release from the system very rapidly, as demonstrated in Figure 5, but they may also be much more strongly attracted to the enzyme, as $\mathrm{MB}$ is. Like $\mathrm{MB}, \mathrm{MO}$ is a planar, multi-ring aromatic dye only $2 \%$ heavier, but negatively charged. It demonstrated no discernable interaction with the enzymes, but partition coefficient trials indicated a partition coefficient for MO between PMMA/DMA hydrogel at pH 3 and the surrounding pH 3 buffer of roughly 500. The sink into which negatively charged solutes release must somehow maintain a low solute concentration in order to effect complete release.

This is demonstrated even more strongly in Figure 7, where the barrier layers now contain $10 \% \mathrm{w} / \mathrm{w} \mathrm{ZnO}$ nanoparticles. Now there is a clear lag between discrete pulses of $\mathrm{MO}$, the first reported example of pulsatile solute release from a BMPR system triggered by glucose. While the first depot exhibits complete release, subsequent pulses from similar depots exhibit progressively less release, with the fourth pulse barely even discernable, consistent with a solute partition coefficient of roughly 500 .

\subsection{BMPR Design.}


In designing a glucose-powered BMPR system, the simplest parameters to vary are the membrane thicknesses, the amount and location of enzyme, and the amount and location of scavenger in the barrier. Figure 8 demonstrates the effect of barrier thickness on release from a $100 \mu \mathrm{m}$ PMMA/DMA depot exposed to a sink with a physiological concentration $(90 \mathrm{mg} / \mathrm{dL})$ of glucose. Without any scavenger in the barrier, acid reaches the depot immediately and begins swelling it, releasing drug. The time at which the depot becomes fully swollen and delaminates from the device (indicated by a sudden increase in release near the top of each curve) increases linearly with barrier thickness. More notably, Figure 8 remains unchanged no matter where the enzyme is located within the barrier. Both of these results indicate that the system is strongly glucose-limited, where glucose flux into the device is the clear rate-limiting step. As the barrier thickness decreases, the distance the glucose must travel decreases and its 

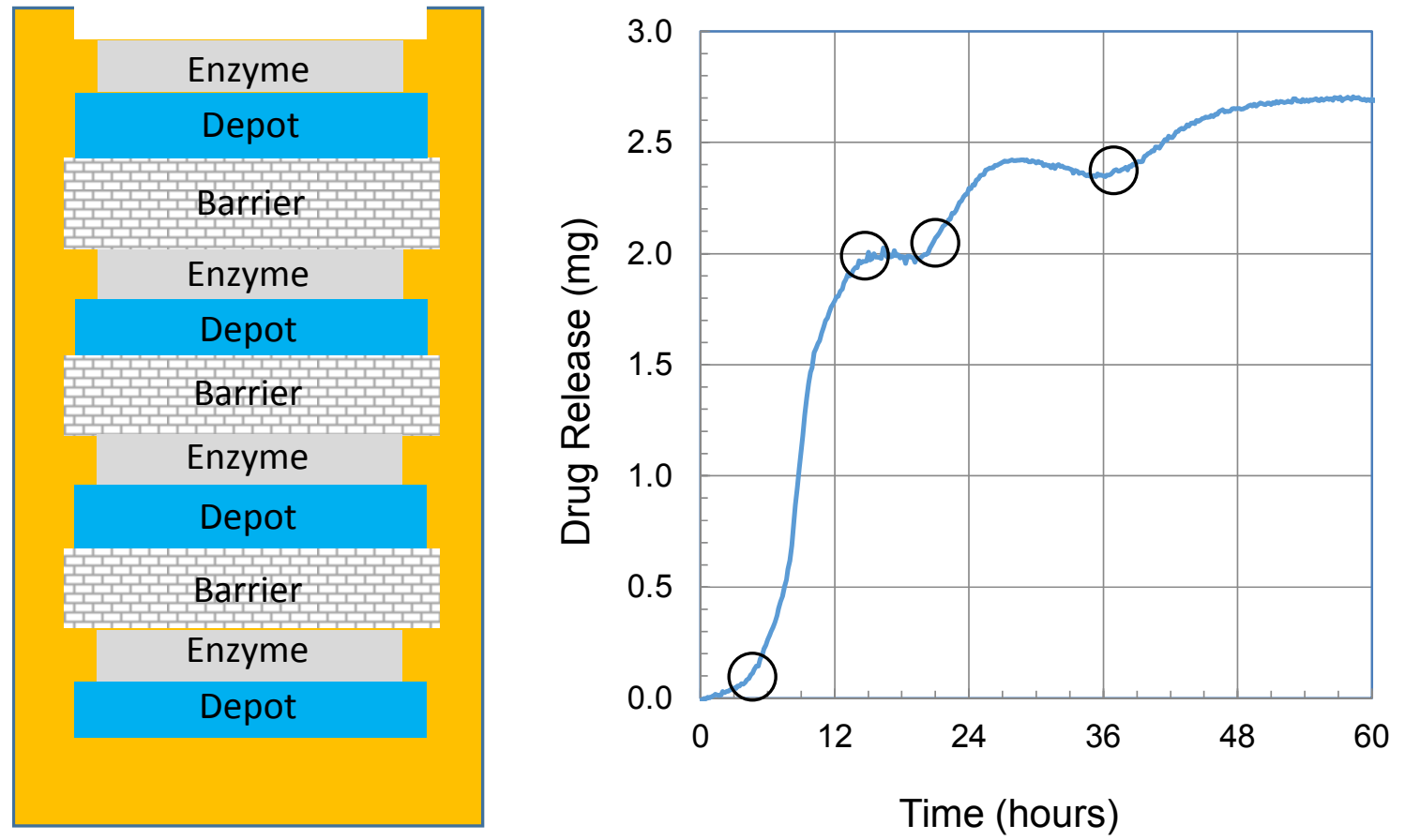

Figure 7: Glucose-powered pulsatile release. At left, schematic of a BMPR devices with ZnO-laden barriers. Though the second depot released prematurely, likely due to poor sidewall adhesion by the first barrier, the chart at right shows the first example of BMPR release using glucose as the stimulant. Subsequent pulses are smalled due to the large partition coefficient of methyl orange in PMMA/DMA. Circles on the chart at right indicate times when depots delaminated from the device. Note that the vertical and horizontal length scales of the schematics are different for clarity. The actual device aspect ratio resembles a coin's.

flux goes up, decreasing the delamination time of the depot proportionally. Similarly, sequestering the enzyme near to the depot requires the glucose to diffuse further before being consumed, decreasing the flux. The flux of acid back towards the sink decreases proportionally, however, canceling the effect of decreased glucose flux perfectly and keeping the delamination time the same regardless of where the enzyme is located. Increasing the amount of enzyme clearly has no effect in this situation, either. Significantly decreasing the amount of enzyme may cause the system to be enzyme limited, at least when the enzyme is not sequestered. This simply prolongs the delamination time further, however. To reduce the delamination time without increasing the external glucose concentration or changing the chemistry of the polymer to swell with less acid, one can decrease the thickness of the barrier, 
as shown in Figure 8, or decrease the thickness of the depot. With half as much depot, delamination occurs twice as fast.

In systems which are limited by enzyme activity rather than glucose concentration, however, the location of the enzyme become much more important, as demonstrated in Figure 9. Here the enzyme activity

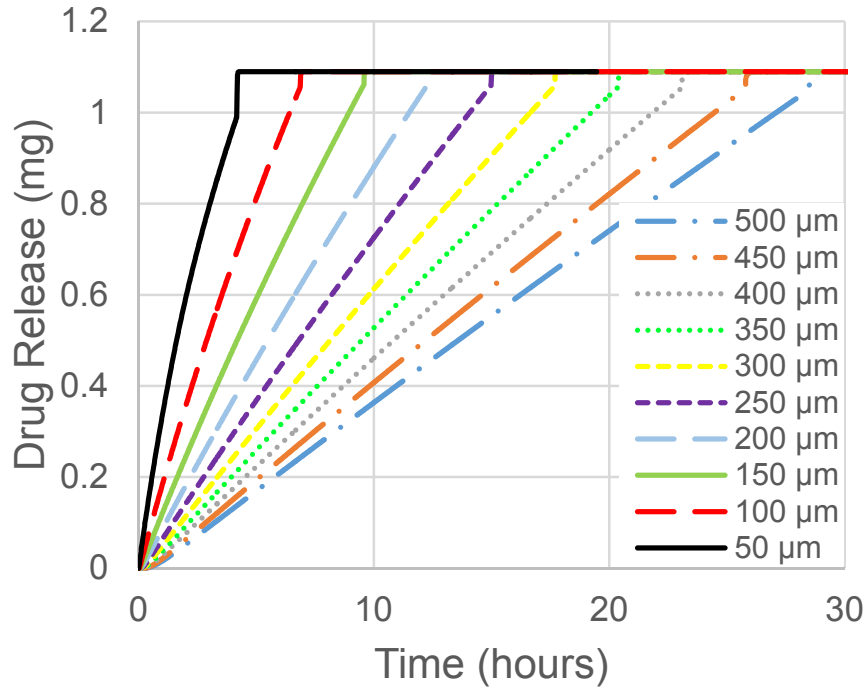

Figure 8: Effect of barrier thickness on depot delamination. Model release profiles demonstrate that in a glucose-limited system, increasing the barrier layer thickness increases the time required to provide sufficient acid to swell the depot, regardless of where the enzyme in the barrier is

concentration is an order of magnitude lower than in Figure 8, and the depot layer thickness has been cut in half to shorten the delamination time. For a $100 \mu \mathrm{m}$ barrier, Figure 9 demonstrates the effect of sequestering the enzyme within various fractions of the barrier nearest to the depot. As the enzyme is sequestered more closely to the depot, the generation of acid remains unchanged since glucose diffuses into the barrier more quickly than it is consumed, and a larger fraction of this acid goes to the depot since it is closer. This results in decreased delamination time due to enzyme sequestration. Decreasing the barrier thickness, on the other hand, actually increases delamination time, as with less membrane there's less enzyme producing acid and less distance for the produced acid to travel to escape the system. The design principles for enzyme-limited systems are opposite those of glucose-limited systems.

A stimulant scavenger, $\mathrm{ZnO}$ in our physical system, provides a delay time regardless of how the system is limited, and if the $\mathrm{ZnO}$ is sequestered at the depot interface, the delay time is the same in both cases. As the center of mass of the $\mathrm{ZnO}$ moves further from the depot 

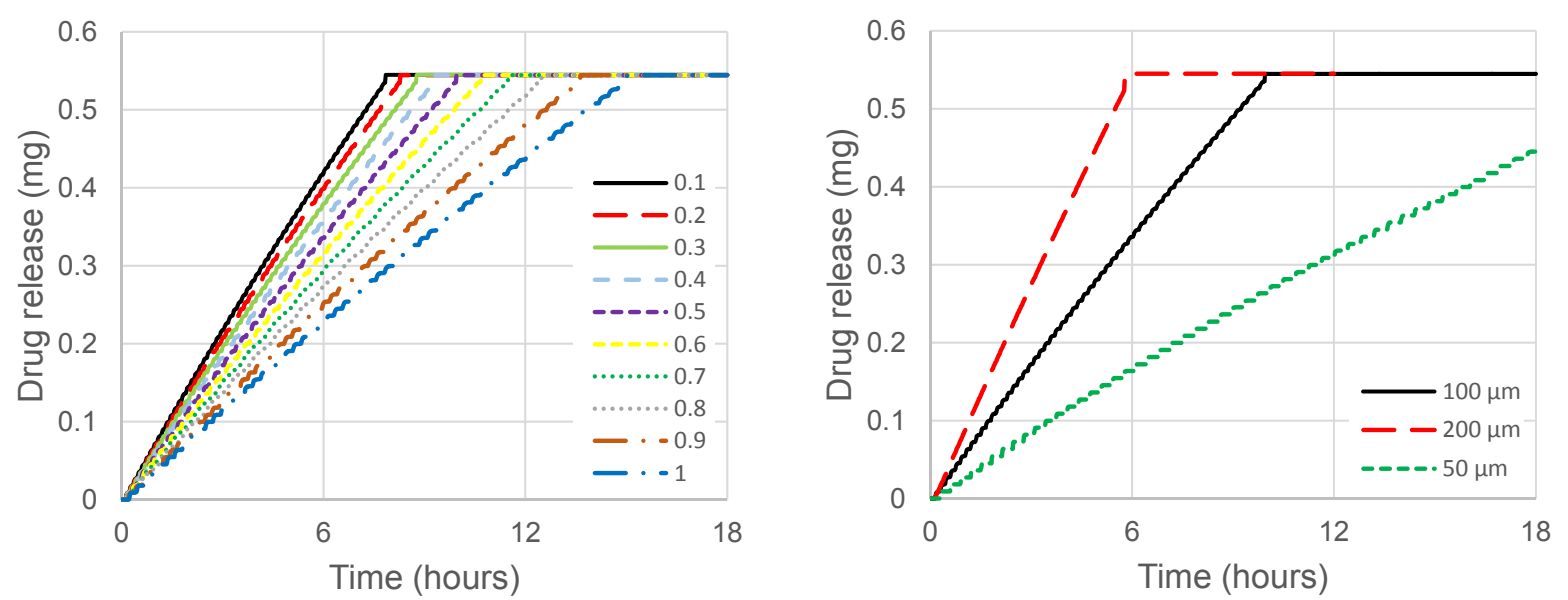

Figure 9: Effect of enzyme sequestration and barrier thickness in enzyme-limited conditions. When glucose is present in excess, the location of the enzyme in the barrier strongly affects the depot delamination time, in the release profiles at left Legend indicates the fraction of the barrier across which the enzyme is distributed, located adjacent to the depot. Chart at right shows that decreasing barrier thickness increases the delamination time of the depot, opposite of the effect in glucose-limited conditions.

interface, however, the delay time it provides decreases, as its closer proximity to generated acid will increase the acid flux consuming it.

\section{Conclusions.}

Pulsatile drug release currently requires either manual application of each pulse or complex electronic and mechanical systems. However, by incorporating common enzymes and acid scavengers into a highly permeable hydrogel, a constant glucose signal can be transduced into a pulsatile acid signal. By incorporating drug into an acid-sensitive polymer, this pulsatile acid signal can be transduced into a pulsatile drug signal. Stacking the two composite membranes together, pulsatile drug release can be obtained from a simple polymer laminate, driven only by a constant sink of water and glucose. This paper provides the first experimental demonstration of such a system, characterizes the system components, models the system computationally, and provides design rubrics for further system development. With sufficient design rubrics and further materials development, the modular nature of the system facilitates rapid point-of-use fabrication of customized drug delivery chips, quickly inserted on an outpatient basis with different drugs and timings for each layer. 


\section{Acknowledgements.}

The authors gratefully acknowledge the National Science Foundation (CBET-1133297) and the American Heart Association (11SDG7600044) for their support of this work. Y.N. participated in this work through a travel grant from the Institute of National Colleges of Technology, Japan (2014-146).

\section{References.}

1. Lévi F, Zidani R, Misset J-L. Randomised multicentre trial of chronotherapy with oxaliplatin, fluorouracil, and folinic acid in metastatic colorectal cancer. The Lancet. 1997;350(9079):681-6.

2. Innominato PF, Roche VP, Palesh OG, Ulusakarya A, Spiegel D, Levi FA. The circadian timing system in clinical oncology. Annals of medicine. 2014;46(4):191-207.

3. McKim WA. Drugs and Behavior. An Introduction to Behavioral Pharmacology. 2007. $416 \mathrm{p}$.

4. Abrams J. A reappraisal of nitrate therapy. Journal of the American Medical Association. 1988;259:396-401.

5. Li JT, Lockey RF, Bernstein IL, Portnoy JM, Nicklas RA. Allergen Immunotherapy: a practice parameter. Annals of Allergy, Asthma \& Immunology. 2003;90(1):1-40.

6. Centers for Disease Control and Prevention. Rabies Vaccine: What You Need to Know. wwwcdcgov/vaccines/pubs/vis/downloads/vis-rabiespdf. 2009.

7. Walters AA, Krastev C, Hill AV, Milicic A. Next generation vaccines: single-dose encapsulated vaccines for improved global immunisation coverage and efficacy. Journal of Pharmacy and Pharmacology. 2015;67(3):400-8.

8. Brabant G, Prank K, Schöfl C. Pulsatile Patterns in Hormone Secretion. Trends in Endocrinology and Metabolism. 1992;3(5):183-90.

9. Hayes FJ, Crowley WFJ. Gonadotropin Pulsations across Development. Hormone Research. 1998;49:163-8.

10. Bhalla AS, Siegel RA. Mechanistic studies of an autonomously pulsing hydrogel/enzyme system for rhythmic hormone delivery. Journal of Controlled Release. 2014;196:261-71.

11. Compston JE. Skeletal actions of intermittent parathyroid hormone: effects on bone remodelling and structure. Bone. 2007;40(6):1447-52. 
12. Jeon JH, Piepgrass WT, Lin Y-L, Thomas MV, Puleo DA. Localized intermittent delivery of simvastatin hydroxyacid stimulates bone formation in rats. Journal of Periodontology. 2008;79(8):1457-64.

13. Richardson TP, Peters MC, Ennett AB, Mooney DJ. Polymeric system for dual growth factor delivery. Nature Biotechnology. 2001;19(11):1029-34.

14. McCoy M. Microencapsulation aids insecticides. Chemical and Engineering News. 2009;87(26):16.

15. Hamilton JG. Needle phobia: a neglected diagnosis. The Journal of family practice. 1995;41(2):169-75.

16. Nir Y, Paz A, Sabo E, Potasman I. Fear of Injections in Young Adults: Prevalence and Associations. American Journal of Tropical Medicine and Hygiene. 2003;68:341-4.

17. Maroni A, Zema L, Del Curto MD, Loreti G, Gazzaniga A. Oral pulsatile delivery: Rationale and chronopharmaceutical formulations. International Journal of Pharmaceutics. 2010;398:1-8.

18. Tang ESK, Chan LW, Heng PWS. Coating of multiparticulates for sustained release. American Journal of Drug Delivery. 2005;3(1):17-28.

19. Thitinan S, McConville JT. Development of a gastroretentive pulsatile drug delivery platform. The Journal of pharmacy and pharmacology. 2012;64(4):505-16.

20. Fujioka K, Sato S, Takada Y, Sasaki Y, Tamura N. Sustained pulsewise release pharmaceutical preparation. JP patent 230654. 1987.

21. Jiang HL, Zhu KJ. Pulsatile protein release from a laminated device comprising of polyanhydrides and $\mathrm{pH}$-sensitive complexes. International Journal of Pharmaceutics. 2000;194:51-60.

22. Jeon JH, Puleo DA. Alternating release of different bioactive molecules from a complexation polymer system. Biomaterials. 2008;29(26):3591-8.

23. Liu X, Pettway GJ, McCauley LK, Ma PX. Pulsatile release of parathyroid hormone from an implantable delivery system. Biomaterials. 2007;28(28):4124-31.

24. Gandhi S, Gosse MD, Nishii Y, Nuxoll E. Barrier-Mediated Pulsatile Release. Journal of Membrane Science. 2015; 495:351-360

25. Gandhi S, Nuxoll E. Non-delaminating Pulsatile Release Composites. Chemical Engineering Science. 2015; submitted, placeholder.

26. Siegel RA, Firestone BA. pH-dependent equilibrium swelling properties of hydrophobic polyelectrolyte copolymer gels. Macromolecules. 1988;21(11):3254-9.

27. Firestone BA, Siegel RA. Kinetics and Mechanisms of Water Sorption in Hydrophobic, Ionizable Copolymer Gels. Journal of Applied Polymer Science. 1991;43:901-14. 
28. Halacheva SS, Adlam DJ, Hendow EK, Freemont TJ, Hoyland J, Saunders BR. Injectable biocompatible and biodegradable $\mathrm{pH}$-responsive hollow particle gels containing poly(acrylic acid): the effect of copolymer composition on gel properties. Biomacromolecules. 2014;15(5):1814-27.

29. Das NK, Mandal BM. Methylene Blue as a Retarder of Free Radical Polymerization: 1. Polymerization of Acrylonitrile, Methyl methacrylate, and Styrene. Polymer. 1982;23(10):1653-8.

30. Nuxoll EE, Cussler EL. The third parameter in reactive barrier films. AIChE Journal. 2005;51(2):456-63.

31. Nuxoll EE, Siegel RA, Cussler EL. Layered reactive barrier films. Journal of Membrane Science. 2005;252(1-2):29-36.

32. Siegel RA, Johannes I, Hunt CA, Firestone BA. Buffer Effects on Swelling Kinetics in Polybasic Gels. Pharmaceutical Research. 1992;9(1):76-81.

33. Liao C, Nicklaus MC. Comparison of Nine Programs Predicting pKa Values of Pharmaceutical Substances. Journal of Chemical Information and Modeling. 2009;49(12):2801-12.

34. Bae YH, Okano T, Kim SW. Temperature Dependence of Swelling of Crosslinked Poly(N,N'-alkyl substituted acrylamides) in Water. Journal of Polymer Science B: Polymer Physics. 1990;28:923-36.

35. Wong F-L, Abdul-Aziz A. Comparative study of poly(vinyl alcohol)-based support materials for the immobilization of glucose oxidase. Journal of Chemical Technology \& Biotechnology. 2008;83(1):41-6.

36. Libby B, Smyrl WH, Cussler EL. Polymer-Zeolite Composite Membranes for Direct Methanol Fuel Cells. AIChE Journal. 2003;49(4):991-1001. 